\title{
Thermal Characterization of Carbon Fiber-Reinforced Carbon Composites
}

\author{
J. D. Macias ${ }^{1}$ • J. Bante-Guerra ${ }^{2}$ • \\ F. Cervantes-Alvarez ${ }^{2}$ - G. Rodrìguez-Gattorno ${ }^{2}$. \\ O. Arés-Muzio ${ }^{2}$ - H. Romero-Paredes ${ }^{1}$. \\ C. A. Arancibia-Bulnes ${ }^{3}$ V. Ramos-Sánchez ${ }^{4}$. \\ H. I. Villafán-Vidales ${ }^{3} \cdot$ J. Ordonez-Miranda ${ }^{5}$. \\ R. Li Voti ${ }^{6}$. J. J. Alvarado-Gil ${ }^{2}$
}

Received: 6 March 2018 / Accepted: 9 April 2018

(C) Springer Science+Business Media B.V., part of Springer Nature 2018

\begin{abstract}
Carbon fiber-reinforced carbon $(\mathrm{C} / \mathrm{C})$ composites consist in a carbon matrix holding carbon or graphite fibers together, whose physical properties are determined not only by those of their individual components, but also by the layer buildup and the material preparation and processing. The complex structure of $\mathrm{C} / \mathrm{C}$ composites along with the fiber orientation provide an effective means for tailoring their mechanical, electrical, and thermal properties. In this work, we use the Laser Flash Technique to measure the thermal diffusivity and thermal conductivity of $\mathrm{C} / \mathrm{C}$ composites made up of laminates of weaved bundles of carbon fibers, forming a regular and repeated orthogonal pattern, embedded in a graphite matrix. Our experimental data show that: i) the cross-plane thermal conductivity remains practically constant around $(5.3 \pm 0.4) \mathrm{W} \cdot \mathrm{m}^{-1} \mathrm{~K}^{-1}$, within the temperature range from $370 \mathrm{~K}$ to
\end{abstract}

J. D. Macias

jdanielmacias@yahoo.es

1 Universidad Autónoma Metropolitana-Iztapalapa, Av. San Rafael Atlixco No. 186, 09340 México D.F, Mexico

2 Departamento de Fisica Aplicada, CINVESTAV-Unidad Merida, Carr. Antigua a Progreso km. 6, 97310 Mérida, Yucatán, Mexico

3 Instituto de Energías Renovables, Universidad Nacional Autónoma de México, Priv. Xochicalco S/N, 62580 Temixco, Morelos, Mexico

4 Facultad de Química, Universidad Autónoma de Chihuahua, Circuito Universitario 8, Campus UACH II, 31125 Chihuahua, Chihuahua, Mexico

5 Institut Pprime, Universite de Poitiers, ISAE-ENSMA, 11 Boulevard Marie et Pierre Curie, Chasseneuil, France

6 Dipartimento di Scienze di Base ed Applicate per l'Ingegneria, Sapienza Universita di Roma, via A. Scarpa 16, 00161 Rome, Italy 
$1700 \mathrm{~K}$. ii) The thermal diffusivity and thermal conductivity along the cross-plane direction to the fibers axis is about five times smaller than the corresponding ones in the laminates plane. iii) The measured cross-plane thermal conductivity is well described by a theoretical model that considers both the conductive and radiative thermal contributions of the effective thermal conductivity.

Keywords $\mathrm{C} / \mathrm{C}$ composite $\cdot$ Anisotropic structures $\cdot$ Solid thermal conductivity $\cdot$ Radiative thermal conductivity $\cdot$ Rosseland extinction coefficient $\cdot$ Solar thermal technology

\section{Introduction}

Carbon fiber-reinforced carbon $(\mathrm{C} / \mathrm{C})$ composites made up of laminates of weaved bundles of carbon fibers embedded in a carbon matrix are well-suited materials for highly demanding applications in which strength, stiffness, and others mechanical properties are critical $[1,2]$. Carbon fibers are long thin filaments mostly composed of carbon atoms that form microscopic crystals (graphitic planes) aligned in parallel along the fiber axis [3, 4]. These graphitic planes exhibit high anisotropy due to the difference between the in-plane and out-of-plane bonding of carbon atoms. This is the reason why the thermophysical properties of carbon fibers along their axes are usually higher than those in the perpendicular radial direction [5, 6]. For instance, the Young modulus, thermal conductivity, and electrical conductivity of carbon fibers along their axes are on the order of $900 \mathrm{GPa}, 1000 \mathrm{~W} \cdot \mathrm{m}^{-1} \mathrm{~K}^{-1}$, and $10^{6} \mathrm{~S} \cdot \mathrm{m}^{-1}$, respectively, which are significantly higher than their corresponding values along their radial direction $[7,8]$. In addition, the degree of mechanical stability of $\mathrm{C} / \mathrm{C}$ composites can be well controlled by the concentration of fibers within to the matrix $[9,10]$. In general, the overall properties of $\mathrm{C} / \mathrm{C}$ composites are determined by the fibers (type, volume fraction, and orientation), the architecture (layer buildup, pores distribution, etc.), and the thermal treatment involving carbonization and graphitization [6]. This latter process is usually applied to improve some of the $\mathrm{C} / \mathrm{C}$ composite properties, such as the thermal diffusivity, electrical conductivity, thermal stability, and resistance to both oxidation and corrosion [11-13]. On the other hand, the impregnation and re-baking processes [14] can be used to change other physical properties, such as the interlaminar shear strength, flexural and tensile strength, bulk density, pore volume, Young's modulus, compressive strength, and resistance to alternating loads, as revealed nondestructively for example with thermal and acoustic methods [15, 16]. The appropriate selection of the production processes and constituent materials allows thus fabricating $\mathrm{C} / \mathrm{C}$ composites with tailored physical and mechanical properties suitable for a wide range of applications [17-20]. High-temperature application of C/C composites includes solar technology [21], furnace construction and heat treatment, internal combustion engines, aerospace technology, among others $[2,22]$. The vibrations of the crystal lattice involving phononphonon, phonon-defect, and phonon-interface interactions are the main source of heat transport in $\mathrm{C} / \mathrm{C}$ composites [23], especially near room temperature. Concerning the phonon-phonon interaction, the mean free path of phonons is very sensitive to temperature [24]. At low temperatures phonons do not scatter frequently with other phonons and defects, and the mean free path is limited mainly by the material boundary. As the temperature reduces the contribution of acoustic phonons to the heat transport becomes more relevant than that of optical ones [25]. On the contrary, as the temperature increases, the phonon-phonon interactions (responsible for the heat conduction) become more frequent, and consequently the 
thermal conductivity of $\mathrm{C} / \mathrm{C}$ composites increases. Also, for temperature $T$ higher than the Debye one, the heat capacity becomes independent of temperature, the number of phonons is proportional to $T$, then thermal conductivity is proportional to mean free path $\lambda_{M F P}$ where one may assume $\lambda_{M F P} \propto T^{-x}$ [26] (in the case of standard graphite, $x$ value is about 0.6 in the range from 800 to $1800 \mathrm{~K}$, see Fig. 3). Thermal properties of C/C composites have been investigated by different researchers: Zhi-Hai Feng et al. [27] described the effects of the composite porosity, the thickness and porosity of the interface phase on the thermal conductivity for $\mathrm{C} /$ C composites with Pitch-Based Matrix. G. Yuan et al. [28] determined the longitudinal thermal conductivity and thermal diffusivity at room temperature of the $\mathrm{C} / \mathrm{C}$ composites, graphitized at $3373 \mathrm{~K}$ and reports the values $896 \mathrm{~W} \cdot \mathrm{m}^{-1} \mathrm{~K}^{-1}$ and $642 \mathrm{~mm}^{2} \mathrm{~s}^{-1}$, respectively, C. W. Ohlhorst et al. [29] examined different $\mathrm{C} / \mathrm{C}$ composites and report in-plane thermal conductivity values from 10 to $233 \mathrm{Wm}^{-1} \mathrm{~K}^{-1}$, whereas through-the-thickness values from 2 to $21 \mathrm{~W} \cdot \mathrm{m}^{-1} \mathrm{~K}^{-1}$, H.B. Shim et al. found that the cross-section type of the reinforcing fibers as well as their orientation, strongly affect the thermal conductivity of carbon fiber-reinforced composites [30], J. Michalowski et al. [31] examined the thermal conductivity of 2D C/C composites, and reported that a thermal treatment, applied after densification, increases the thermal conductivity of the composite. Crystallization of carbon matrix material produces this effect.

In this work, we report the thermal diffusivity and thermal conductivity of $\mathrm{C} / \mathrm{C}$ composites measured in the perpendicular direction to the fibers axes, through the Laser Flash Technique (LFT) [32], for temperatures from $300 \mathrm{~K}$ to $1700 \mathrm{~K}$. Taking into account the anisotropy of the $\mathrm{C} / \mathrm{C}$ composites, both thermal properties have also been measured by means of a calorimetric method [33], in three different directions along the plane of the fiber laminates at $308 \mathrm{~K}$. Furthermore, the response of our samples to solar radiation and high temperatures has been assessed by measuring their solar absorptance and thermal emittance in the UV-VIS-NIR-MIR wavelength range.

\section{Materials and Methods}

Our $\mathrm{C} / \mathrm{C}$ composite consisting in laminates of weaved bundles of carbon fibers, forming a regular and repeated orthogonal pattern within a carbon matrix, are shown in Fig. 1. The mass density, fiber volume fraction, and porosity of samples are about $(1.51 \pm 0.01) \mathrm{g} \cdot \mathrm{cm}^{-3}, 60 \%$, and $8 \%$, respectively [34]. All samples have been darkened with a homogeneous thin layer of graphite (GRAPHIT 33, CRC Industries Europe NV), in order to ensure the uniform conversion of a laser beam light into heat on the illuminated sample surface, to measure their thermal diffusivity [35] by Laser Flash Technique (LFT). The working principle of this standard transient technique, consists in illuminating one side of a sample by impinging a laser pulse (in our experiments: pulse length and beam spot diameter were $1 \mathrm{~ms}$ and $6 \mathrm{~mm}$, respectively) and recording the temperature rise of the opposite side [36] as a function of time, using an infrared detector located just in front of the sample [37]. The sample thermal diffusivity is then obtained by fitting a proper theoretical model to this temperature rise. The simplest model for describing the transient behavior of temperature was proposed by Parker et al. [32], who considered one-dimensional heat transfer with adiabatic boundary condition [38]. Parker's model is only valid for homogeneous samples supporting a predominant one-dimensional heat flux with negligible heat losses [39]. As the temperature increases, this latter assumption is difficult to fulfill, given that the radial heat losses becomes more important [40], especially for thicker samples. Under these conditions, Parker et al. [32] model has to be properly extended 
$[41,42]$. Thermal diffusivity experimental data analysis were performed taking into account corrections models for adiabatic [43], finite pulse [42, 44-47] and lateral heat losses [40, 48]. In order to obtain the thermal diffusivity as a function of temperature $(D(T))$ of our $\mathrm{C} / \mathrm{C}$ samples, in the direction perpendicular to the plane of laminates, we prepared circular samples ( $25.2 \mathrm{~mm}$ in diameter and $1.5 \pm 0.2 \mathrm{~mm}$ in thickness) and then were measured by LFT. In the same fashion, to determine the thermal diffusivity in the directions parallel and perpendicular to the plane of laminate (Fig. 1), we cut rectangular rods of $\mathrm{C} / \mathrm{C}$ composites $(30.2 \times 9.6 \times$ $5.2 \mathrm{~mm}$ ), and we applied the modified Angstrom method [33]. This method consists in the periodically heating one end of a bar and monitoring the phase lag of the temperature field at its opposite end, phase lag value is related with the thermal diffusivity [49]. To calculate the heat capacity of the $\mathrm{C} / \mathrm{C}$ composites samples, we implemented an indirect method, where the maximum of the temperature raise in the sample is compared to the maximum of the temperature raise of the reference sample. If we assume that the light into heat energy conversion and the sample reflectance are the same for sample and reference, then both, the $\mathrm{C} / \mathrm{C}$ composite and the reference sample (standard graphite) are measured under the same conditions [50] so the energy of the laser pulse and the sensitivity of the infrared detector are the same for both measurements. Since the energy supply to the sample (sam) and the reference (ref), is the same, the heat capacity of the sample can be calculated by means of the following relation:

$$
c_{\text {sam }}=\frac{c_{r e f} \Delta T_{r e f} m_{r e f}}{\Delta T_{\text {sam }} m_{\text {sam }}}
$$

where $\Delta T$ is the temperature raise $(\mathrm{K}), m$ is the mass $(\mathrm{g})$ and $c$ is the heat capacity $\left(\mathrm{J} \cdot \mathrm{g}^{-1} \mathrm{~K}^{-1}\right)$. Once the thermal diffusivity and heat capacity have been obtained, the thermal conductivity $\kappa(T)$ of the sample with mass density $\rho(T)$ can be obtained from the relationship: $\kappa(T)=$ $D(T) c(T) \rho(T)$.

Reflectance spectroscopy is a powerful tool for characterizing the optical properties of a great diversity of materials applied in solar technology [51-54]. The analysis can be done in two wavelength ranges of interest: Ultraviolet/visible/near infrared and medium infrared, where solar spectrum radiation wavelengths and infrared emission range are located respectively. Spectral (near-normal) directional/hemispherical reflectance $R(\lambda)$ values were obtained with commercial spectrophotometers equipped with integrating sphere [55], in the range from 300 to $1000 \mathrm{~nm}$ we used an AVASpec2048 spectrophotometer, and in the range from 1000 to

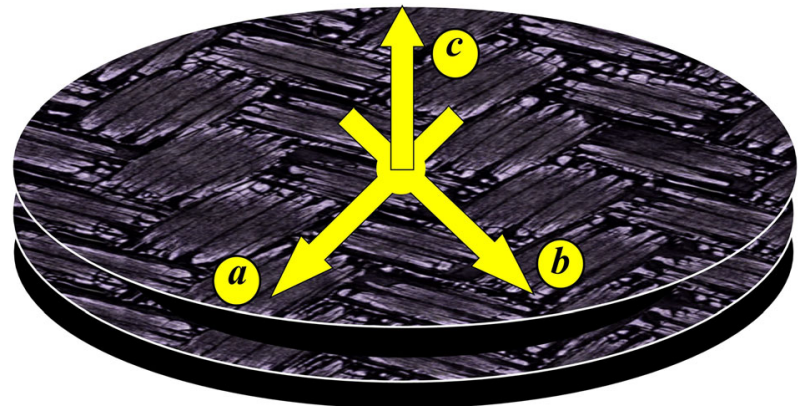

Fig. $1 \mathrm{C} / \mathrm{C}$ composite made up of laminates with weaved bundles (tows) of carbon fibers forming a regular and repeated orthogonally pattern, embedded in a graphite matrix. In-plane measurement direction corresponds to $a$ and $b$ arrows, whereas $c$ indicates out-plane measurement direction 
$2000 \mathrm{~nm}$ an AvaSpec-NIR256-2.5 spectrophotometer was used. In both cases, measurements were performed with an integrating sphere 50-LS-HAL, in which the angle of incidence of the light is near-normal to the sample surface. Likewise, $R(\lambda)$ data in the range of 2 to $15 \mu \mathrm{m}$ were obtained with a FTIR spectrophotometer Perkin Elmer Frontier NIR/MIR equipped with an integrating sphere Pico IntegratIR. Solar absorbance $(\alpha)$ and thermal emittance $(\varepsilon(T))$ can be calculated with the following relations [56]:

$$
\alpha=\frac{\int_{\lambda 1}^{\lambda 2} I_{S U N}(\lambda)[1-R(\lambda)] d \lambda}{\int_{\lambda 1}^{\lambda 2} I_{S U N}(\lambda) d \lambda}, \varepsilon(T)=\frac{\int_{\lambda 3}^{\lambda 4} I_{B B}(\lambda, T)[1-R(\lambda)] d \lambda}{\int_{\lambda 3}^{\lambda 4} I_{B B}(\lambda, T) d \lambda}
$$

where $I_{S U N}(\lambda)$ is the solar irradiance spectrum, and $I_{B B}(\lambda, T)$ is the black body radiation function.

\section{Results}

Figure 2 shows the values of the thermal diffusivity, measured perpendicular to the plane of the laminate (through-thickness direction), and heat capacity in our $\mathrm{C} / \mathrm{C}$ composites in the temperature range from 300 to $1700 \mathrm{~K}$ with the LFT. Values obtained are consistent with those are reported in literature [29].

Materials with anisotropic structures such as $\mathrm{C} / \mathrm{C}$ composite have different linear expansion coefficients in different directions [25]. As result of this, the total volumetric expansion is distributed unequally among the three axes, and bulk density can change as function of temperature. Bulk density as function of temperature was determined by using the relation $\rho(T)=\rho_{O} /\left(0.9965-1.32 \times 10^{-5} T+4.58 \times 10^{-8} T^{2}-4.98 \times 10^{-11} T^{3}\right)\left[\mathrm{g} \cdot \mathrm{cm}^{-3}\right]$ [57], being $\rho_{O}$ the density value at $298 \mathrm{~K}$. The effective cross-plane thermal conductivity $\kappa_{\text {eff }}$ of our $\mathrm{C} / \mathrm{C}$ composites as a function of temperature is determined by the sum of the phonon (solid) and photon (radiative) contributions along with the convective one, due to the heat transfer through the gas present in the pores. This latter component is usually much smaller than the other two

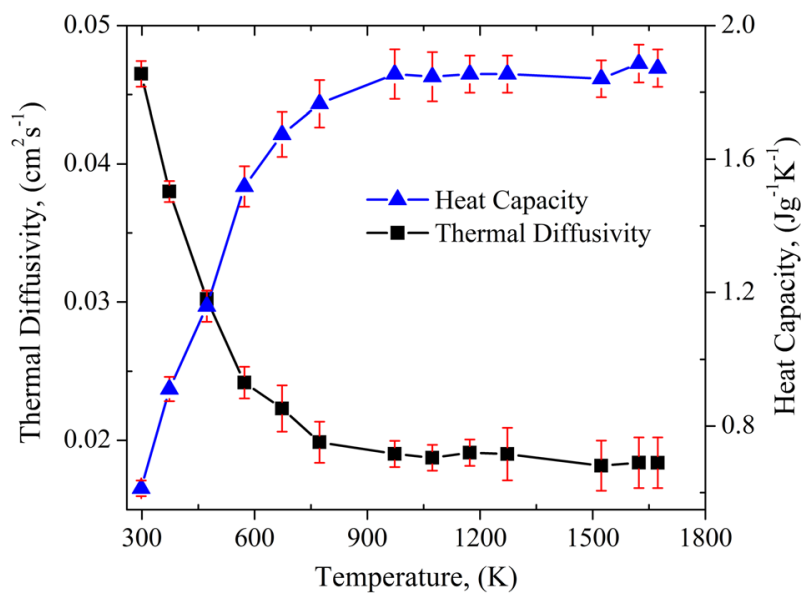

Fig. 2 Measured cross-plane thermal diffusivity and heat capacity of our $\mathrm{C} / \mathrm{C}$ samples in the temperature range from $300 \mathrm{~K}$ to $1700 \mathrm{~K}$ 
ones [58] and therefore it will be neglected in the present work. Under this assumption, $\kappa_{\text {eff }}$ is given by (see appendix Eq. 14):

$$
\kappa_{e f f}(T)=(1-p)^{2} \kappa_{m e}(T)\left[\gamma(T)+\frac{1}{2} \beta(T)^{2}+\beta(T) \sqrt{\gamma(T)+\frac{\beta(T)^{2}}{4}}\right]+\frac{16}{3} \sigma_{S B} \frac{n^{2}(T)}{\sigma_{e}(T)} T^{3}
$$

Where all parameters are defined in the Appendix. Figure 3 shows the experimental values of the thermal conductivity measured along the cross-plane direction in comparison with the fitted predictions of Eq. 3 for a large range of temperatures. The good agreement between the experimental and theoretical values of $\kappa_{\text {eff }}(T)$ improves for temperatures higher than $750 \mathrm{~K}$, because the used data for a single fiber thermal conductivity is not enough accurate (Eq. 12), in the temperature range below $750 \mathrm{~K}$. Also, one can observe that the thermal conductivity of $\mathrm{C} /$ $\mathrm{C}$ composites samples reaches an almost constant value of $(5.3 \pm 0.4) \mathrm{W} \cdot \mathrm{m}^{-1} \mathrm{~K}^{-1}$, in the temperature range from $370 \mathrm{~K}$ to $1700 \mathrm{~K}$. This behavior indicates that the increase of the radiative component of the thermal conductivity is compensated with the decrease of the conductive one, as the sample temperature increases.

The thermal diffusivity $D$ of our $\mathrm{C} / \mathrm{C}$ composites has been measured in three directions; $a$ and $b$ in the plane of laminate, and $c$ perpendicular to the plane of laminate (Fig. 1) at $308 \mathrm{~K}$. On purpose, we apply a periodic heating [49] $(f=0.048 \mathrm{~Hz}$. $)$ at one end of samples and retrieve $D$ by means of the modified Angstrom method [33]. Results for $D$ and $\kappa$ are summarized in Table 1 . We did not find significant differences in thermal diffusivity values along the $a$ and $b$ directions, since the fiber counts in the warp and weft directions are equivalent and that hence there is no preferential presence of empty interspace among fibers in either direction. The thermal conductivity is thus invariant in the plane of the weave [59]. By contrast, the out-plane ( $c$ direction) thermal diffusivity are one order of magnitude smaller than the corresponding inplane measurements. This latter fact indicates that there is a strong anisotropy on the sample's heat conduction, such that the heat flows better in the laminated planes than across them

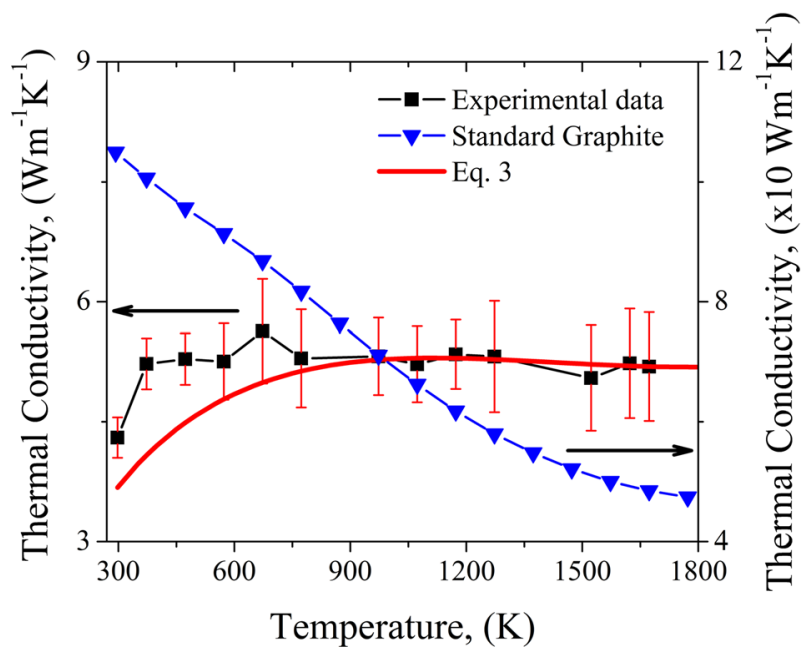

Fig. 3 Experimental values measured for the cross-plane thermal conductivity of our $\mathrm{C} / \mathrm{C}$ composites in comparison with the fitted predictions of Eq. (3). The thermal conductivity remains almost constant around the value $(5.3 \pm 0.4) \mathrm{W} \cdot \mathrm{m}^{-1} \mathrm{~K}^{-1}$, within the temperature range from $370 \mathrm{~K}$ to $1700 \mathrm{~K}$ 
Table 1 Thermal diffusivity and thermal conductivity of our $\mathrm{C} / \mathrm{C}$ composite at $308 \mathrm{~K}$, measured in three directions: two parallel to the plane of the laminate ( $a$ and $b$ in Fig. 1) and another one perpendicular to this plane ( $c$ direction) The average values of the heat capacity and mass density were $(0.65 \pm 0.02) \mathrm{J} \cdot \mathrm{g}^{-1} \mathrm{~K}^{-1}$ and $(1.51 \pm 0.01)$ $\mathrm{g} \cdot \mathrm{cm}^{-3}$, respectively.

\begin{tabular}{llll}
\hline Direction of measurement & Sample thickness $(\mathrm{mm})$ & $\begin{array}{l}\text { Thermal diffusivity } \\
\left(\times 10^{-2} \mathrm{~cm}^{2} \cdot \mathrm{s}^{-1}\right)\end{array}$ & $\begin{array}{l}\text { Thermal conductivity } \\
\left(\mathrm{W} \cdot \mathrm{m}^{-1} \mathrm{~K}^{-1}\right)\end{array}$ \\
\hline$a$ (in-plane) & $30.1 \pm 0.1$ & $14.8 \pm 0.9$ & $14.5 \pm 1.5$ \\
$b$ (in-plane) & $30.2 \pm 0.1$ & $15.1 \pm 1.1$ & $14.8 \pm 1.7$ \\
$c$ (out-plane) & $5.2 \pm 0.1$ & $2.6 \pm 0.1$ & $2.6 \pm 0.2$ \\
\hline
\end{tabular}

(perpendicular to the planes). The large number of long fibers provide thus the continuous path for the phonon transmission, which is attenuated along their radial $c$ direction [25]. The thermal conductivity measured in the direction parallel to the laminate plane is about five times greater than that along the plane perpendicular to the laminate, which agrees with the results reported in literature [25, 29, 60-63].

The reflectance spectrum of our $\mathrm{C} / \mathrm{C}$ composites measured for wavelengths ranging from 0.3 to $15 \mu \mathrm{m}$ along with the solar irradiance spectrum [64] are shown in Fig. 4. Based on these spectra, Eq. (2) yields a solar absorbance and thermal emittance of $\alpha=(78 \pm 2) \%$ and $\varepsilon(T)=(59 \pm 1) \%$, respectively at $373 \mathrm{~K}$. Given that the determination of the spectral selectivity $\eta$ through the simplest relation $\eta=\alpha / \varepsilon(T)$ [65-68]. However, mistakes can be made in the calculation of the spectral selectivity, for example: a solar absorber with $=70$ $(\alpha=0.7$ and $\varepsilon(T)=0.01)$, apparently has a better performance than a solar absorber with $\eta=32(\alpha=0.98$ and $\varepsilon(T)=0.03)$ which is not true, because high absorption at low wavelengths has a stronger impact than the low emittance at longer wavelengths [69]. Z. Chen et al. [70] propose a more accurate way to calculate $\eta$ considering that solar absorptance is twice as important as the thermal emittance. In this work we have estimated $\eta$ with the relation $\eta=\alpha-0.5 \varepsilon(T)$, which yields $\eta=48.5 \%$. In high solar concentration systems (600 suns or more) the influence of the thermal emittance is less important and it becomes almost independent of temperature [71]. The inset in Fig. 4 shows the thermal emittance (Eq.2) calculated as function of the temperature of a black body in the range from 373 to $3073 \mathrm{~K}$.

\section{Conclusions}

We have determined the thermal diffusivity and thermal conductivity of carbon fiberreinforced carbon $(\mathrm{C} / \mathrm{C})$ composites along their cross-plane and in-plane directions. We have found that the thermal diffusivity and thermal conductivity along the cross-plane direction to the fibers axis is about five times smaller than the corresponding ones in the laminates plane. The cross-plane thermal conductivity measured as a function of temperature has been well described by a theoretical model that considers both the conductive and radiative thermal contributions of the effective thermal conductivity. We have found that the cross-plane thermal conductivity remains practically constant around $(5.3 \pm 0.4) \mathrm{W} \cdot \mathrm{m}^{-1} \mathrm{~K}^{-1}$, within the temperature range from $300 \mathrm{~K}$ to $1700 \mathrm{~K}$. Furthermore, we have determined an average spectral selectivity $\eta=48.5 \%$ which could be a very useful parameter in applications of solar thermal technology. 


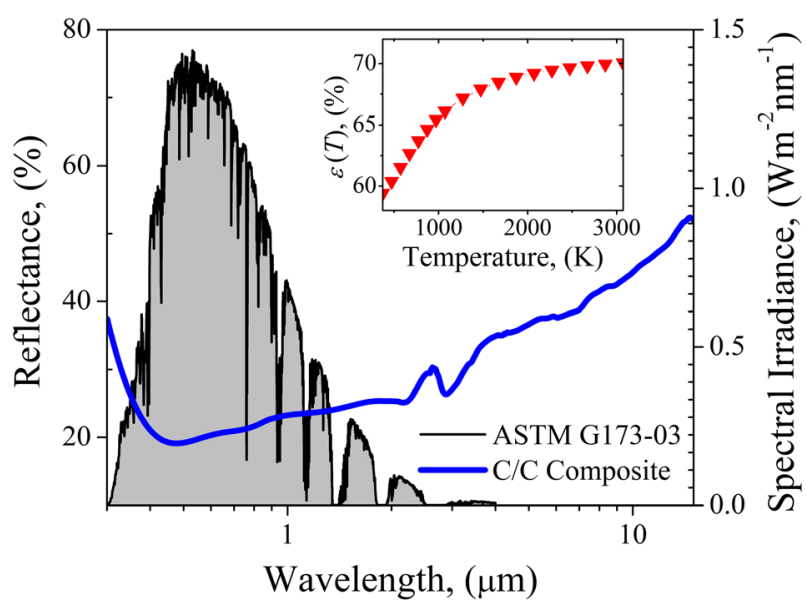

Fig. 4 Reflectance spectrum of our $\mathrm{C} / \mathrm{C}$ composites measured for wavelengths ranging from $0.3 \mu \mathrm{m}$ to $15 \mu \mathrm{m}$, along with the sun radiation spectrum ASTM G173-03 [64]. The inset figure corresponds to the thermal emittance (Eq.2) calculated as function of the temperature of a black body in the range from 373 to $3000 \mathrm{~K}$

Acknowledgements Authors acknowledge the financial support received by the "Fondo Sectorial ConacytSecretaría de Energía-Sustentabilidad Energética and the "Centro Mexicano de Innovación en Energía Solar (CeMIESol)" Grant no. 207450 within the Strategic Project No. 10 "Combustibles Solares y Procesos Industriales (COSOL-pi)", as well as to the Instituto de Energías Renovables of the Universidad Nacional Autónoma de México, Universidad Autónoma Metropolitana (Unidad Iztapalapa), Universidad Autónoma de Chihuahua and to the Centro de Investigacion y de Estudios Avanzados del Instituto Politecnico Nacional (CINVESTAV), for their assistance with financial and strategic management. This work was also partially supported by Projects 192 "Fronteras de la ciencia" and 251882 "Investigación Científica Básica 2015". Additionally, Juan Daniel Macias acknowledges the financial support received by the "Fondo Sectorial Conacyt-Secretaría de EnergíaSustentabilidad Energética" under the program: "Estancias Posdoctorales en Mexico".

\section{Appendix}

\section{Thermal conductivity modeling}

According to diverse theoretical models reported in the literature, the effective thermal conductivity $\kappa_{e f f}(T)$ of $\mathrm{C} / \mathrm{C}$ composites can be expressed in terms of the thermal conductivity of the fibers and matrix, fibers volume fraction [72], fibers mass fraction [30], graphitic planes direction [12], contact heat transfer rate among fibers [19], and the effective contact area between two plates [5]. Considering the thermal and geometrical properties of the individual components, here we model $\kappa_{e f f}(T)$ as the sum of its solid conductive $\left(\left(\kappa_{c}(T)\right)\right.$ and radiative $\left(\left(\kappa_{r}(T)\right)\right.$ contributions, as follows

$$
\kappa_{e f f}(T)=\kappa_{c}(T)+\kappa_{r}(T)
$$

Concerning the conductive contribution, for a $\mathrm{C} / \mathrm{C}$ composite made up of laminate bundles of fibers forming a regular orthogonal pattern and uniform delamination between fiber mats embedded in a solid matrix, as shown in Fig. 5, the in-plane $\left(\kappa_{\|}\right)$and out-plane $\left(\kappa_{\perp}\right)$ solid thermal conductivities of this bidirectional composite are given by:

$$
\kappa_{\|}=n_{v} \kappa_{v \|}(\theta)+n_{h} \kappa_{h \|}(\theta)
$$




$$
\kappa_{\perp}=\kappa_{1 \perp}
$$

where $\kappa_{1 \perp}$ is the composite thermal conductivity perpendicular to unidirectional fibers (see Fig. 5), $n_{v}$ and $n_{h}$ are the number fractions of fibers in the vertical and horizontal directions (with $\left.n_{v}+n_{h}=1\right)$ and $\kappa_{v \|}(\theta)$ and $\kappa_{h \|}(\theta)$ are the in-plane thermal conductivity of vertical and horizontal unidirectional fibers in a direction making an angle $\theta$ with the vertical axis calculated as follows [59]:

$$
\begin{gathered}
\kappa_{v \|}(\theta)=\kappa_{1 \|} \cos ^{2}(\theta)+\kappa_{1 \perp} \sin ^{2}(\theta) \\
\kappa_{h \|}(\theta)=\kappa_{1 \perp} \cos ^{2}(\theta)+\kappa_{1 \|} \sin ^{2}(\theta)
\end{gathered}
$$

Where $\kappa_{1 \|}$ is the composite thermal conductivity parallel to unidirectional fibers, as shown in Fig. 5, considering that the numbers of fibers in vertical and horizontal directions in Fig. 5 are equal $\left(n_{v}=n_{h}=1 / 2\right)$, Eqs. (5a), (6a) and (6b) yields to:

$$
\kappa_{\|}=\frac{1}{2}\left(\kappa_{1 \|}+\kappa_{1 \perp}\right)
$$

Equation (7) explicitly shows that the in-plane thermal conductivity of the composite in Fig. 5 is independent of the direction in which is measured, as was first predicted by Pilling et al. [72].
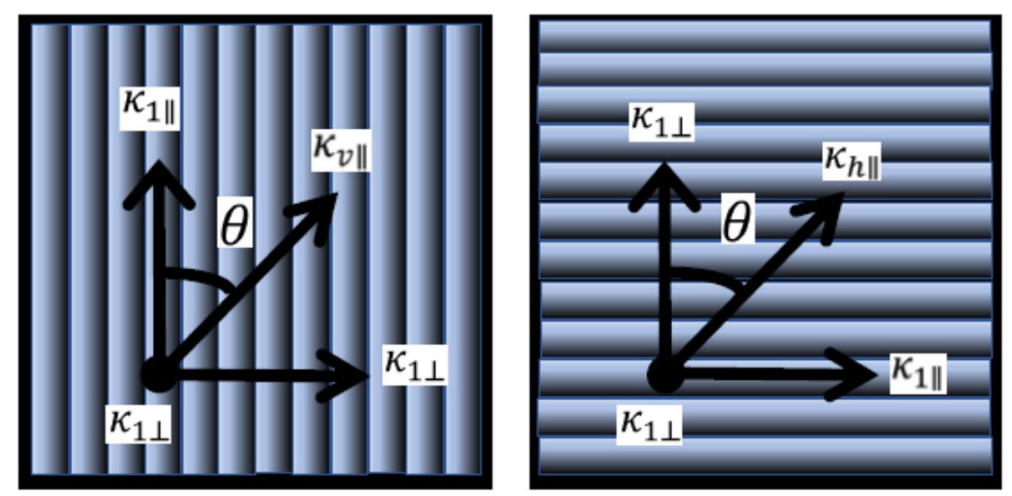

Fig. 5 Thermal conductivity components parallel and perpendicular to unidirectional fibers

The thermal conductivity $\kappa_{1 \|}$ of the unidirectional composite in Fig. 6 is simply given by the series model, as follows [73]:

$$
\kappa_{1 \|}=f \kappa_{f \|}+(1-f-p) \kappa_{m \|}
$$

where $f$ and $p$ are the respective volume fractions of the fibers and pores, while $\kappa_{f \|}$ and $\kappa_{m \|}$ are the fiber and matrix thermal conductivities in the axial direction of the fibers, respectively. 

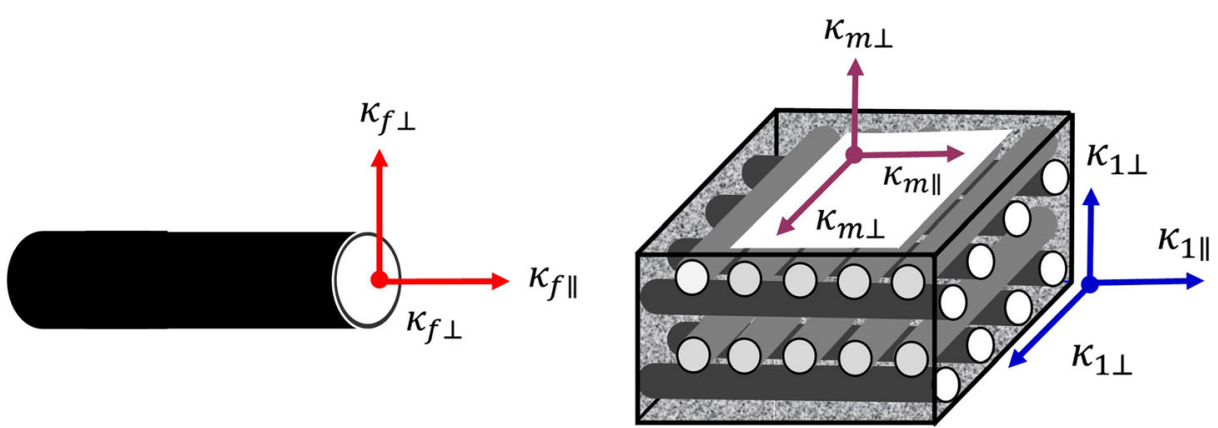

Fig. 6 Thermal conductivity components of a single fiber (Left) and considering a 3D C/C composite unit cell (Right)

On the other hand, the out-plane thermal conductivity $\kappa_{1 \perp}$ can be determined by means of the Bruggeman's model [74] applied to cylindrical particles [75], as follows:

$$
\begin{gathered}
\frac{\kappa}{\kappa_{m}}=a+\frac{1}{2} \beta^{2}+\beta \sqrt{a+\frac{\beta^{2}}{4}} \\
\beta=(1-a)(1-v) \\
\alpha=\left(\frac{\kappa_{m}}{\kappa_{p}}+\frac{a_{\kappa}}{a_{f}}\right)^{-1}
\end{gathered}
$$

where $\kappa_{m}$ is the thermal conductivity of the continuous matrix and $\kappa_{p}$ is the thermal conductivity of air inside the pores or the porous filler (fibers in this case), and they interact with an interface thermal resistance $R$, being $a_{\kappa}=R \kappa_{m}$ the Kapitza radius [76], $a_{f}$ the average radius of the fillers of volume fraction $v$ and $\kappa$ is the overall composite thermal conductivity. Considering the fibers and matrix like an effective matrix with cylindrical pores represented by the fibers (see Fig. 7), if we take $\kappa_{m}=\kappa_{1 \perp}(p=0)$, and $\kappa_{p}=0$ (air thermal conductivity), $R \rightarrow \infty$ and $v=p$ in Eqs. (9a) to (9c), which render:

$$
\kappa_{1 \perp}=(1-p)^{2} \kappa_{m}
$$

Equation (10) indicates that the presence of pores reduces the effective thermal conductivity of the matrix and fibers by a factor of $(1-p)^{2}$. The thermal conductivity $\kappa_{1 \perp}(p=0)$ can now be calculated by reusing Eqs. (9a) to (9c) with $\kappa_{m}=\kappa_{m \perp}, \kappa_{p}=\kappa_{f \perp}$ and $v=f /(1-p)$ being the volume fraction of fibers in the solid matrix alone. After making these substitutions in Eqs. (9a) to (9c) and combining the result with Eq. (10), one obtains:

$$
k_{1 \perp}=(1-p)^{2} k_{m \perp}\left(a_{\perp}+\frac{1}{2} \beta_{\perp}^{2}+\beta_{\perp} \sqrt{a_{\perp}+\frac{\beta_{\perp}^{2}}{4}}\right)
$$

where $\beta_{\perp}$ and $a_{\perp}$ are defined by Eqs. (9b) and (9c), for $\kappa_{m \perp}, \kappa_{f \perp}$ and $v=f /(1-p)$. Eq. (11) considers the effects of the radius $a$ and volume fraction $f$ of the fibers, as well as of the 
porosity $p$ and the interface thermal resistance $R$. The thermal conductivities $\kappa_{1 \|}$ and $\kappa_{1 \perp}$ of a composite with unidirectional fibers thus determine the overall thermal conductivities $\kappa_{\perp}$ and $\kappa_{\|}$of the bidirectional composite shown in Fig. 5.

Fig. 7 Matrix and fibers are considered like an effective matrix with cylindrical pores, represented by the fibers

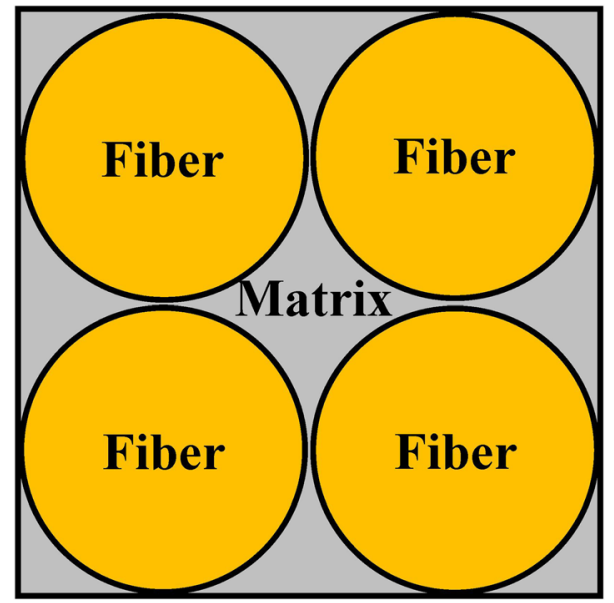

\section{In-plane and out-plane thermal conductivities of a single carbon fiber}

To calculate the thermal conductivity as a function of the temperature of the $\mathrm{C} / \mathrm{C}$ composites (Eq. 11), it is necessary knowing the data of the axial and transverse thermal conductivities of a single carbon fiber and the pure matrix (without carbon fibers). The effective transverse thermal conductivity of the pitch-based carbon fibers, was predicted by H.S Huang et al. [77]. They calculated the in-plane and out-plane thermal conductivity for straight graphite sheets. The value of the simulated equivalent transverse thermal conductivity was $50.8 \mathrm{~W} \cdot \mathrm{m}^{-1} \mathrm{~K}^{-1}$ for perfect fibers and the measured value for real fibers was $12 \mathrm{Wm}^{-1} \mathrm{~K}^{-1}$. Therefore, we can take the value of 27 for the anisotropy factor $\left(\kappa_{\|} / \kappa_{\perp}\right)$, a value which is consistent with those reported in literature [3, 62]. We can also have assumed that this factor does not change significantly as function of temperature [30]. C. Pradere et al. [78] report the longitudinal thermal conductivity in the temperature range from 750 to $2000 \mathrm{~K}$, of single carbon fibers made with the same raw materials and exposed to a heat treatment similar those $\mathrm{C} / \mathrm{C}$ composites examined in this work. Out of plane thermal conductivity of the fibers in the temperature range from 750 to $2000 \mathrm{~K}$ can be adjusted to the expression [78]:

$$
\kappa_{P}(T)=5.5045+4.97 \times 10^{-3} T-4.78 \times 10^{-6} T^{2}+1.20 \times 10^{-9} T^{3}
$$

\section{Pure matrix thermal conductivity}

There are different and varied methods to build $\mathrm{C} / \mathrm{C}$ composites, usually these methods consist on impregnation of sheets of carbon fiber fabrics with precursors (i.e. phenol-formaldehyde resins or gas phase precursors such as propane) that give rise the matrix of the composite [79]. Once the sheets are impregnated, the composites are molded and cured, subsequently they are carbonized at $1273 \mathrm{~K}$ under vacuum. The processes of impregnation and carbonization are 
repeated several times to change the density and porosity of the composite. Under these conditions the thermal conductivity $\kappa_{C}$ of the composite obeys the simple mixing rule for two phases system: $\kappa_{c}=\kappa_{f} V_{f}+\kappa_{m} V_{m}, V$ is the volume fraction, and $c, f$, and $m$ refer to composite, filler and matrix, respectively. Due to $\kappa_{f} V_{f} \ggg \kappa_{m} V_{m}$, then $\kappa_{c} \sim \kappa_{f} V_{f}$. However, when the $\mathrm{C} / \mathrm{C}$ composites are subjected to a high temperature treatment (HTT) at temperatures above $2273 \mathrm{~K}$, the experimentally thermal conductivity value of the composite increases significantly with respect to that calculated from the simple mixing rule $[12,24]$. In addition, it has been demonstrated that after several HTT cycles, the thermal conductivity value of the $\mathrm{C} / \mathrm{C}$ composite is no longer affected. Due to the fact that the thermal conductivity of the single fibers does not change significantly during the HTT cycles of $\mathrm{C} / \mathrm{C}$ composites, because the fibers were previously subjected to a similar HTT and their structural properties such as crystallinity, crystalline distribution, molecular orientation, carbon content, and the amount of defects, are well formed [6]. Then, an increase in the thermal conductivity of the $\mathrm{C} / \mathrm{C}$ composite can be attributed to changes in the crystallographic parameters of carbon matrix (interlayer spacing $d_{002}$, and stack height $L_{\mathrm{c}}$ ) [31]. For all the above, $\mathrm{C} / \mathrm{C}$ composites with same fiber fabric and matrix precursors but subjected to different HTT may have different thermal conductivities [80], and this behavior is most noticeable at high temperature. One way to determine and to distinguish the effect of components in the $\mathrm{C} / \mathrm{C}$ composite (carbon fibers and matrix), is by obtaining the thermal conductivity of samples of pure matrix (without carbon fibers). A good approximation to thermal behavior of the pure matrix is to consider it as glasslike carbon (GC) material [31], then the effective thermal conductivity of the matrix $\left(\kappa_{m e}\right)$ can be expressed by: $\kappa_{m e}=\xi \kappa_{G C}$, where $\xi$ is a constant that depends on volume fraction of solid matrix and porous, as well as the pore shape factor [81]. Effective thermal conductivity of the matrix out of plane can be expressed as [50]:

$$
\kappa_{m e}(T)=-0.2199+0.0123 T-1.08 \times 10^{-5} T^{5}+4.46 \times 10^{-9} T^{3}-7.12 \times 10^{-13} T^{4}
$$

Solid thermal conductivity as a function of the temperature $\kappa_{c}(T)$ of the $\mathrm{C} / \mathrm{C}$ composites (Eq. 11) can be rewritten as:

$$
\kappa_{c}(T)=(1-p)^{2} \kappa_{m e}(T)\left[\alpha(T)+\frac{1}{2} \beta(T)^{2}+\beta(T) \sqrt{\alpha(T)+\frac{\beta(T)^{2}}{4}}\right]
$$

\section{Radiative thermal conductivity}

Effective thermal conductivity in porous materials can be expressed in terms of the solid thermal conductivity and the radiative conductivity (thermal conduction by gas convection is usually ignored due to its low value with respect to the other heat transfer mechanisms). Radiative conductivity $\left(\kappa_{r}(T)\right)$ is based on Rosseland diffusion approximation [82], and it can be expressed by [83]:

$$
\kappa_{r}(T)=\frac{16}{3} \sigma_{S B} \frac{n^{2}(T)}{\sigma_{e}(T)} T^{3}
$$

Where $\sigma_{e}(T)$ is the Rosseland mean extinction coefficient [84] which is an average value of the spectral extinction coefficient $\left(\sigma_{e, \nu}(T)\right)$ weighted by the local spectral energy flux, $\sigma_{S B}$ is the 
Stefan-Boltzmann's constant $\left(5.67 \times 10^{-8} \mathrm{~W} \cdot \mathrm{m}^{-2} \mathrm{~K}^{-4}\right)$, and $n$ is the effective refractive index ( $n=1$ in this work). The Rosseland mean extinction coefficient is defined as follows:

$$
\begin{gathered}
\frac{1}{\sigma_{e}(T)}=\frac{\int_{0}^{\infty} \frac{1}{\sigma_{e, \nu}(T)} \frac{\partial I(T, \nu)}{\partial T} d \nu}{\int_{0}^{\infty} \frac{\partial I(T, \nu)}{\partial T} d \nu} \\
\frac{\partial I(T, \nu)}{\partial T}=\frac{2 h^{2} \nu^{4}}{\kappa_{B} T^{2} c^{2}} \frac{e^{\frac{h \nu}{\kappa_{B} T}}}{\left(e^{\frac{h \nu}{\kappa_{B} T}}-1\right)^{2}}
\end{gathered}
$$

Where $\partial I(T, \nu) / \partial T$ is the temperature derivative of Planck's equation. For homogeneous media, and considering $\sigma_{e, \nu}(T)$ independent on thickness, according to Beer's law:

$$
\sigma_{e, \nu}(T) d=-\ln \left[\tau_{n, \nu}(T)\right]
$$

Being $d$ the thickness of the sample and $\tau_{n, \lambda}(T)$ is the spectral transmittance percentage, which could be measured with a Fourier transform infrared (FTIR) spectrometer. Figure 8 shows the spectral transmittance percentage $\left(\tau_{n, \lambda}(T)\right)$ for two samples $(600$ and $800 \pm 30 \mu \mathrm{m}$ in thickness) obtained with a FTIR spectrometer, and the Rosseland mean extinction coefficient $\left(\sigma_{e}(T)\right)$ calculated with Eq. (16a).

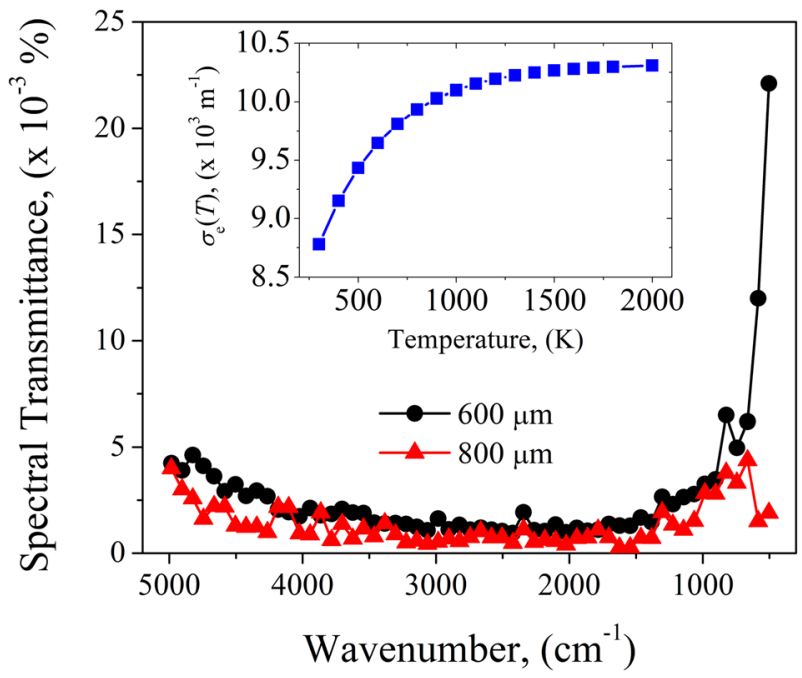

Fig. 8 Spectral transmittance percentage $\left(\tau_{n, \lambda}(T)\right)$ obtained by FTIR spectrometry for two samples $(600$ and $800 \pm 30 \mu \mathrm{m}$ in thickness). The inset graph corresponds to Rosseland mean extinction coefficient $\left(\sigma_{e}(T)\right)$ calculated as function of temperature (Eq. 16a)

Figure 9 shows the effective thermal conductivity (Eq. 4), the solid thermal conductivity (Eq. 14) and radiative conductivity (Eq. 15) as function of temperature. The effective thermal 
conductivity of the $\mathrm{C} / \mathrm{C}$ composite for temperatures below $1000 \mathrm{~K}$ is dominated mainly by solid thermal conductivity (99.4\%). However, for temperatures above $1000 \mathrm{~K}$, the contribution of radiative conductivity increases appreciably until reaching the value of $4.5 \%$ of the effective conductivity of the $\mathrm{C} / \mathrm{C}$ composite at $2000 \mathrm{~K}$.

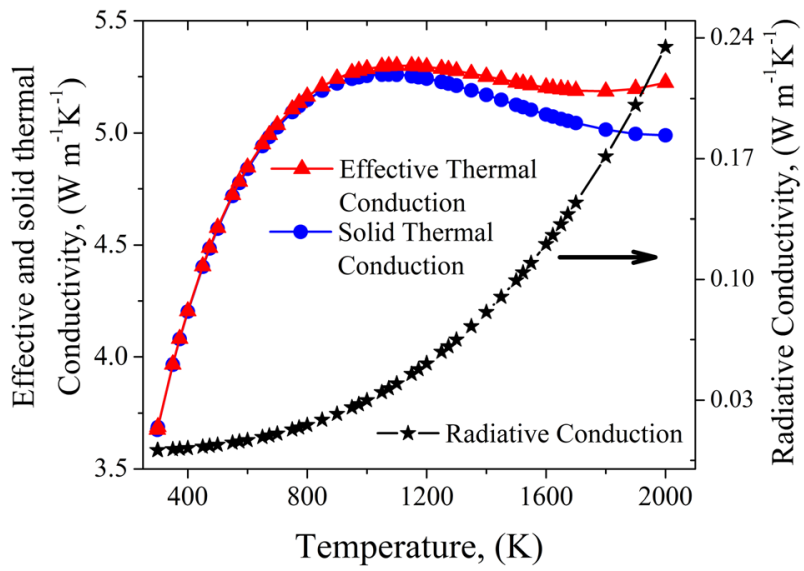

Fig. 9 Effective thermal conductivity obtained from Eq. 4, the solid thermal conductivity based on Eq. 14, and radiative conductivity determined using Eq. 15 as function of temperature

\section{References}

1. Chand, S.: Carbon fibers for composites. J. Mater. Sci. 35(6), 1303-1313 (2000)

2. Krenkel, W., Berndt, F.: C/C-SiC composites for space applications and advanced friction systems. Mater. Sci. Eng. A. 412(1-2), 177-181 (2005)

3. Tian, T., Cole, K.D.: Anisotropic thermal conductivity measurement of carbon-fiber/epoxy composite materials. Int. J. Heat Mass Transf. 55(23-24), 6530-6537 (2012)

4. Sauder, C., Lamon, J., Pailler, R.: The tensile behavior of carbon fibers at high temperatures up to $2400{ }^{\circ} \mathrm{C}$. Carbon. 42(4), 715-725 (2004)

5. Pedraza, D.F., Klemens, P.G.: Effective conductivity of polycrystalline graphite. Carbon. 31(6), 951-956 (1993)

6. Huang, X.: Fabrication and properties of carbon fibers. Materials (Basel). 2(4), 2369-2403 (2009)

7. Minus, M.L., Kumar, S.: The processing, properties, and structure of carbon fibers. JOM J. Miner. Met. Mater. Soc. 57, 52-58 (2005)

8. Ghosh, S., Calizo, I., Teweldebrhan, D., Pokatilov, E.P., Nika, D.L., Balandin, A.A., Bao, W., Miao, F., Lau, C.N.: Extremely high thermal conductivity of graphene: prospects for thermal management applications in nanoelectronic circuits. Appl. Phys. Lett. 92(15), 151911 (2008)

9. Fu, S.Y., Lauke, B., Mäder, E., Yue, C.Y., Hu, X.: Tensile properties of short-glass-fiber-and short-carbonfiber-reinforced polypropylene composites. Compos. Part A Appl. Sci. Manuf. 31(10), 1117-1125 (2000)

10. Fu, S.Y., Lauke, B., Mäder, E., Hu, X., Yue, C.Y.: Fracture resistance of short-glass-fiber-reinforced and short-carbon-fiber-reinforced polypropylene under Charpy impact load and its dependence on processing. Mater. Process. Technol. 89-90, 501-507 (1999)

11. Qiu, L., Zheng, X.H., Zhu, J., Su, G.P., Tang, D.W.: The effect of grain size on the lattice thermal conductivity of an individual polyacrylonitrile-based carbon fiber. Carbon. 51(1), 265-273 (2013)

12. Newcomb, B.A.: Processing, structure, and properties of carbon fibers. Compos. Part A Appl. Sci. Manuf. 91, 262-282 (2016)

13. Jacobson, N., Roth, D., Rauser, R., Cawley, J., Curry, D.: Oxidation of carbon/carbon through coating cracks. ECS Trans. 16(44), 125-135 (2009)

14. Manocha Lalit, M.: High performance carbon-carbon composites. Sadhana. 28(1-2), 349-358 (2003) 
15. Dehoux, T., Wright, O.B., Li Voti, R.: Picosecond time scale imaging of mechanical contacts. Ultrasonics. 50(2), 197-201 (2010)

16. Steinberger, R., Valadas-Leitao, T., Ladstatter, E., Pinter, G., Billinger, W., Lang, R.: Infrared thermographic techniques for non-destructive damage characterization of carbon fibre reinforced polymers during tensile fatigue testing. Int. J. Fatigue. 28(10), 1340-1347 (2006)

17. Thostenson, E.T., Li, W.Z., Wang, D.Z., Ren, Z.F., Chou, T.W.: Carbon nanotube/carbon fiber hybrid multiscale composites. J. Appl. Phys. 91(9), 6034-6037 (2002)

18. Zhang, Z., Wu, Z., Sun, Y., Li, F.: Design aspects of the Chinese modular high-temperature gas-cooled reactor HTR-PM. Nucl. Eng. Des. 236(5-6), 485-490 (2006)

19. Fukai, J., Kanou, M., Kodama, Y., Miyatake, O.: Thermal conductivity enhancement of energy storage media using carbon fibers. Energy Convers. Manag. 41(14), 1543-1556 (2000)

20. Balat-Pichelin, M., Hernandez, D., Olalde, G., Rivoire, B., Robert, J.F.: Concentrated solar energy as a diagnostic tool to study materials under extreme conditions. J. Sol. Energy Eng. 124(3), 215 (2002)

21. Loutzenhiser, P.G., Meier, A., Steinfeld, A.: Review of the two-step H2O/CO2-splitting solar thermochemical cycle based on $\mathrm{Zn} / \mathrm{ZnO}$ redox reactions. Materials (Basel). 3(11), 4922-4938 (2010)

22. Lin, T., Jia, D., He, P., Wang, M., Liang, D.: Effects of fiber length on mechanical properties and fracture behavior of short carbon fiber reinforced geopolymer matrix composites. Mater. Sci. Eng. A. 497(1-2), 181-185 (2008)

23. Burger, N., Laachachi, A., Ferriol, M., Lutz, M., Toniazzo, V., Ruch, D.: Review of thermal conductivity in composites: mechanisms, parameters and theory. Prog. Polym. Sci. 61, 1-28 (2016)

24. Heremans, J., Rahim, I., Dresselhaus, M.S.: Thermal conductivity and Raman spectra of carbon fibers. Phys. Rev. B. 32(10), 6742-6747 (1985)

25. Luo, R., Liu, T., Li, J., Zhang, H., Chen, Z., Tian, G.: Thermophysical properties of carbon/carbon composites and physical mechanism of thermal expansion and thermal conductivity. Carbon. 42(14), 2887-2895 (2004)

26. Gonnet, P.: Thermal Conductivity and Coefficients of Thermal Expansion of SWNTs/Epoxy Nanocomposites. The Florida State University (2004)

27. Feng, Z.H., Zhi, J.Y., Fan, Z., Sun, D., Si, C., Wang, X.-D.: An analytical model of thermal conductivity for carbon/carbon composites with pitch-based matrix. Adv. Mech. Eng. 7(1), 242586 (2015)

28. Yuan, G., Li, X., Dong, Z., Xiong, X., Rand, B., Cui, Z., Cong, Y., Zhang, J., Li, Y., Zhang, Z., Wang, J.: Pitch-based ribbon-shaped carbon-fiber-reinforced one-dimensional carbon/carbon composites with ultrahigh thermal conductivity. Carbon. 68, 413-425 (2014)

29. Ohlhorst, C.W., Vaughn, W.L., Ransone, P.O., Tsou, H.T.: Thermal conductivity database of various structural carbon-carbon composite materials. NASA Tech. Memo. 4787, (1997)

30. Shim, H.B., Seo, M.K., Park, S.J.: Thermal conductivity and mechanical properties of various cross-section types carbon fiber-reinforced composites. J. Mater. Sci. 37(9), 1881-1885 (2002)

31. Michalowski, J., Mikociak, D., Konsztowicz, K.J., Blazewicz, S.: Thermal conductivity of 2D C-C composites with pyrolytic and glass-like carbon matrices. J. Nucl. Mater. 393(1), 47-53 (2009)

32. Parker, W.J., Jenkins, R.J., Butler, C.P., Abbott, G.L.: Flash method of determining thermal diffusivity, heat capacity, and thermal conductivity. J. Appl. Phys. 32(9), 1679-1684 (1961)

33. Nunes dos Santos, W., Nicolau dos Santos, J., Mummery, P., Wallwork, A.: Thermal diffusivity of polymers by modified angström method. Polym. Test. 29(1), 107-112 (2010)

34. Schunk Kohlenstofftechnik GmbH: Schunk-Carbon fiber-reinforced carbon (C/C). [Online]. Available: www.schunk-group.com

35. Kim, S.K., Kim, Y.J.: Determination of apparent thickness of graphite coating in flash method. Thermochim. Acta. 468(1-2), 6-9 (2008)

36. Shibata, H., Suzuki, A., Ohta, H.: Measurement of thermal transport properties for molten silicate glasses at high temperatures by means of a novel laser flash technique. Mater. Trans. 46(8), 1877-1881 (2005)

37. Nishi, T., Shibata, H., Ohta, H., Waseda, Y.: Thermal conductivities of molten Iron, cobalt, and nickel by laser flash method. Metall. Mater. Trans. A. 34, 2801-2807 (2003)

38. Dos Santos, W.N., Mummery, P., Wallwork, A.: Thermal diffusivity of polymers by the laser flash technique. Polym. Test. 24(5), 628-634 (2005)

39. Min, S., Blumm, J., Lindemann, A.: A new laser flash system for measurement of the thermophysical properties. Thermochim. Acta. 455(1-2), 46-49 (2007)

40. Clark III, L.M., Taylor, R.E.: Radiation loss in the flash method for thermal diffusivity. J. Appl. Phys. 46(2), 714-719 (1975)

41. Cowan, R.D.: Pulse method of measuring thermal diffusivity at high temperatures. J. Appl. Phys. 34(4), 926-927 (1963)

42. Cape, J.A., Lehman, G.W.: Temperature and finite pulse-time effects in the flash method for measuring thermal diffusivity. J. Appl. Phys. 34(7), 1909-1913 (1963) 
43. Sheindlin, M., Halton, D., Musella, M., Ronchi, C.: Advances in the use of laser-flash techniques for thermal diffusivity measurement. Rev. Sci. Instrum. 69(3), 1426-1436 (1998)

44. Azumi, T., Takahashi, Y.: Novel finite pulse-width correction in flash thermal diffusivity measurement. Rev. Sci. Instrum. 52(9), 1411-1413 (1981)

45. Takahashi, Y.: Measurement of thermophysical properties of metals and ceramics by the laser-flash method. Int. J. Thermophys. 5(1), 41-52 (1984)

46. Lechner, T., Hahne, E.: Finite pulse time effects in flash diffusivity measurements. Thermochim. Acta. 218, 341-350 (1993)

47. Dusza, L.: Combined solution of the simultaneous heat loss and finite pulse corrections with the laser flash method. High Temp.-High Press. 27-28, 467-473 (1995)

48. Mendioroz, A., Fuente-Dacal, R., Apiñaniz, E., Salazar, A.: Thermal diffusivity measurements of thin plates and filaments using lock-in thermography. Rev. Sci. Instrum. 80(7), 74904 (2009)

49. Bodzenta, J., Burak, B., Nowak, M., Pyka, M., Szalajko, M., Tanasiewicz, M.: Measurement of the thermal diffusivity of dental filling materials using modified Angstrom's method. Dent. Mater. 22(7), 617-621 (2006)

50. K. Shinzato and T. Baba, "A Laser Flash Apparatus for thermal diffusivity and specific heat capacity measurements,” J. Therm. Anal. Calorim., vol. 64, pp. 413-422, 2001

51. Zhang, Q.C.: Recent progress in high-temperature solar selective coatings. Sol. Energy Mater. Sol. Cells. 62(1), 63-74 (2000)

52. Gelin, K., Boström, T., Wäckelgård, E.: Thermal emittance of sputter deposited infrared reflectors in spectrally selective tandem solar absorbers. Sol. Energy. 77(1), 115-119 (2004)

53. Yue, S., Yueyan, S., Fengchun, W.: High-temperature optical properties and stability of AlxOy-AlNx-Al solar selective absorbing surface prepared by DC magnetron reactive sputtering. Sol. Energy Mater. Sol. Cells. 77(4), 393-403 (2003)

54. Rebouta, L., Pitães, A., Andritschky, M., Capela, P., Cerqueira, M.F., Matilainen, A., Pischow, K.: Optical characterization of TiAlN/TiAlON/SiO2 absorber for solar selective applications. Surf. Coatings Technol. 211, 41-44 (2012)

55. Carlsson, B., Möller, K., Köhl, M., Frei, U., Brunold, S.: Qualification test procedure for solar absorber surface durability. Sol. Energy Mater. Sol. Cells. 61(3), 255-275 (2000)

56. Duffie, J.A., Beckman, W.A.: Solar Engineering of Thermal Processes, 4th Edit edn. John Wiley \& Sons, New Jersey (2013)

57. Whittaker, A.J., Taylor, R., Tawil, H.: Thermal transport properties of carbon-carbon fibre composites I. Thermal diffusivity measurements. Proc. R. Soc. Lond. A. 430, 167-181 (1990)

58. Baxter, R., Rawlings, R., Iwashita, N., Sawada, Y.: Effect of chemical vapor infiltration on erosion and thermal properties of porous carbon/carbon composite thermal insulation. Carbon. 38(3), 441-449 (2000)

59. Carslaw, H.S., Jaeger, J.C.: Conduction of Heat in Solids, 2nd edn. Oxford University Press, London (1964)

60. Sun, K., Stroscio, M.A., Dutta, M.: Graphite C-axis thermal conductivity. Superlattice. Microst. 45(2), 6064 (2009)

61. Manocha, L.M., Warrier, A., Manocha, S., Sathiyamoorthy, D., Banerjee, D.: Thermophysical properties of densified pitch based carbon/carbon materials-I. Unidirectional composites. Carbon. 44, 480-487 (2006)

62. Bertolotti, M., Ferrari, A., Liakhou, G.L., Li Voti, R., Marras, A., Ezquerra, T.A., Balta-Calleja, F.J.: Thermal anisotropy of polymer carbon fiber composites as revealed by photodeflection methods. J. Appl. Phys. 78(9), 5706-5712 (1995)

63. Leahu, G., Li Voti, R., Larciprete, M.C., Sibilia, C., Bertolotti, M., Nefedov, I., Anoshkin, I.V.: Thermal characterization of carbon nanotubes by Photothermal techniques. Int. J. Thermophys. 36(5-6), 13491357 (2015)

64. ASTM G173-03(2012): Standard Tables for Reference Solar Spectral Irradiances: Direct Normal and Hemispherical on $37^{\circ}$ Tilted Surface, ASTM International, West Conshohocken, PA (2012)

65. Leon, M.A., Kumar, S.: Mathematical modeling and thermal performance analysis of unglazed transpired solar collectors. Sol. Energy. 81(1), 62-75 (2007)

66. Russell, F.: Heat transfer analysis and modeling of a parabolic trough solar receiver implemented in engineering equation solver. Natl. Renew. Energy Lab., Report No. NREL/TP-550-34169 (2003)

67. Ho, C.K., Pacheco, J.E.: Derivation of a Levelized cost of coating (LCOC) metric for evaluation of solar selective absorber materials. Energy Procedia. 69, 415-423 (2015)

68. Kalogirou, S.A.: Solar thermal collectors and applications. Prog. Energy Combust. Sci. 30(3), 231-295 (2004)

69. Burlafinger, K., Vetter, A., Brabec, C.J.: Maximizing concentrated solar power (CSP) plant overall efficiencies by using spectral selective absorbers at optimal operation temperatures. Sol. Energy. 120, 428-438 (2015) 
70. Chen, Z., Boström, T., Nguyen, Q.: Carbon nanotube spectrally selective solar absorbers. Proceedings of the EuroSun 2014 Conference, pp. 1-7 (2015)

71. Moon, J., Kim, T.K., VanSaders, B., Choi, C., Liu, Z., Jin, S., Chen, R.: Black oxide nanoparticles as durable solar absorbing material for high-temperature concentrating solar power system. Sol. Energy Mater. Sol. Cells. 134, 417-424 (2015)

72. Pilling, M.W., Yates, B., Black, M.A., Tattersall, P.: The thermal conductivity of carbon fibre-reinforced composites. J. Mater. Sci. 14(6), 1326-1338 (1979)

73. Nan, C.W., Birringer, R., Clarke, D.R., Gleiter, H.: Effective thermal conductivity of particulate composites with interfacial thermal resistance. J. Appl. Phys. 81(10), 6692-6699 (1997)

74. Bruggemann, D.A.G.: Berechnung verschiedener physikalischer Konstanten von heterogenen substanzen. Ann. Phys. 24, 636-679 (1935)

75. Ordonez-Miranda, J., Alvarado-Gil, J.J.: Thermal conductivity of nanocomposites with high volume fractions of particles. Compos. Sci. Technol. 72(7), 853-857 (2012)

76. Nan, C.W., Liu, G., Lin, Y., Li, M.: Interface effect on thermal conductivity of carbon nanotube composites. Appl. Phys. Lett. 85(16), 3549-3551 (2004)

77. Huang, H.S., Ganguli, S., Roy, A.K.: Prediction of the transverse thermal conductivity of pitch-based carbon fibers. J. Compos. Mater. 48(11), 1383-1390 (2013)

78. Pradere, C., Batsale, J.C., Goyheneche, J.M., Pailler, R., Dilhaire, S.: Thermal properties of carbon fibers at very high temperature. Carbon. 47(3), 737-743 (2009)

79. Jie, C., Xiang, X., Peng, X.: Thermal conductivity of unidirectional carbon/carbon composites with different carbon matrixes. Mater. Des. 30(4), 1413-1416 (2009)

80. Feng, Z., Fan, Z., Kong, Q., Xiong, X., Huang, B.: Effect of high temperature treatment on the structure and thermal conductivity of 2D carbon/carbon composites with a high thermal conductivity. New Carbon Mater. 29(5), 357-362 (2014)

81. Guan, K., Wu, J., Cheng, L.: Modeling of thermal conductivity of CVI-densified composites at fiber and bundle level. Materials (Basel). 9(12), (2016)

82. Spinnler, M., Winter, E.R.F., Viskanta, R.: Studies on high-temperature multilayer thermal insulations. Int. J. Heat Mass Transf. 47(6-7), 1305-1312 (2004)

83. Gong, P., Buahom, P., Tran, M.P., Saniei, M., Park, C.B., Pötschke, P.: Heat transfer in microcellular polystyrene/multi-walled carbon nanotube nanocomposite foams. Carbon. 93, 819-829 (2015)

84. Zhao, J.J., Duan, Y.Y., Wang, X.D., Wang, B.X.: Experimental and analytical analyses of the thermal conductivities and high-temperature characteristics of silica aerogels based on microstructures. J. Phys. D. Appl. Phys. 46(1), 15304 (2013) 